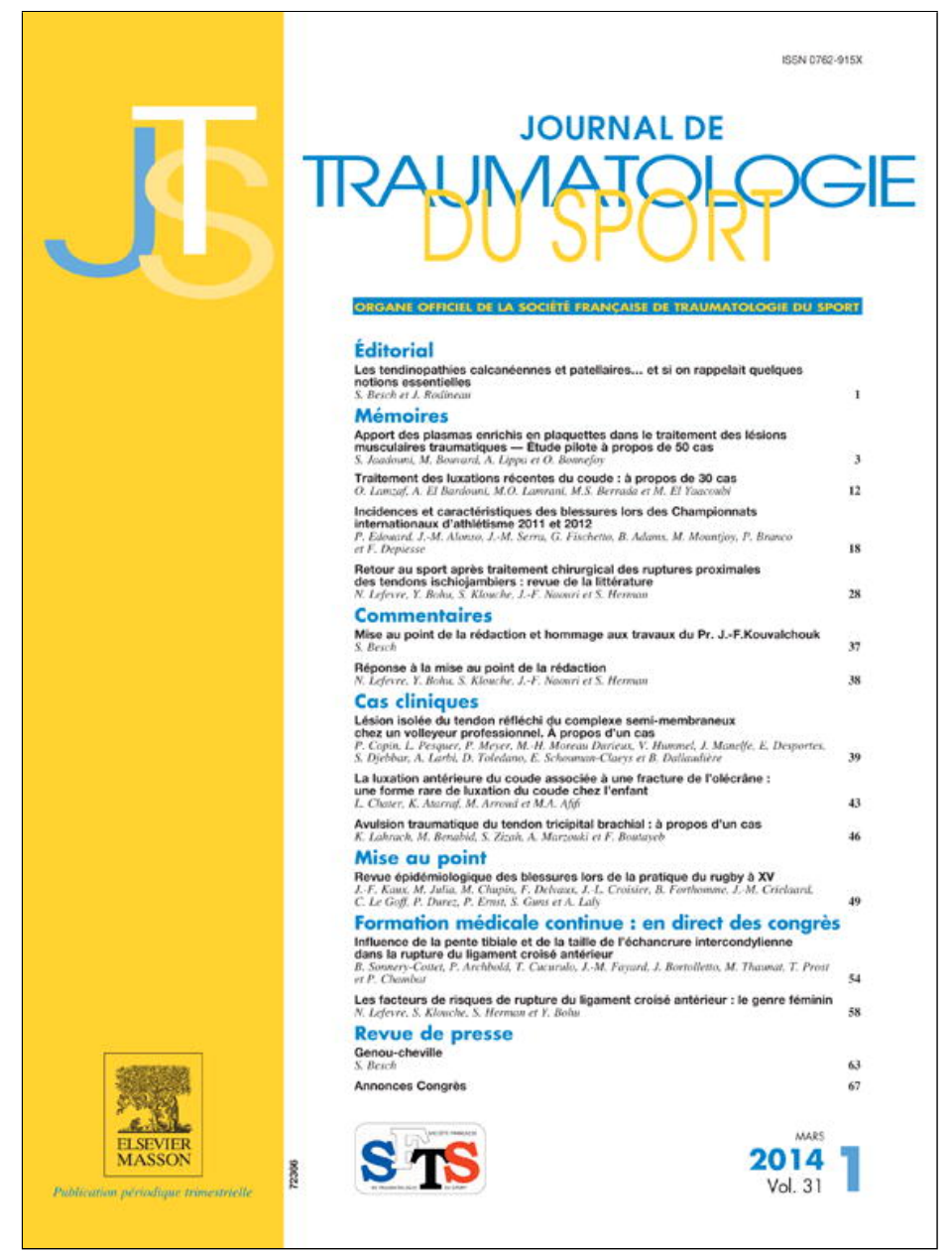

This article appeared in a journal published by Elsevier. The attached copy is furnished to the author for internal non-commercial research and education use, including for instruction at the authors institution and sharing with colleagues.

Other uses, including reproduction and distribution, or selling or licensing copies, or posting to personal, institutional or third party websites are prohibited.

In most cases authors are permitted to post their version of the article (e.g. in Word or Tex form) to their personal website or institutional repository. Authors requiring further information regarding Elsevier's archiving and manuscript policies are encouraged to visit:

http://www.elsevier.com/authorsrights 
Mise au point

\title{
Revue épidémiologique des blessures lors de la pratique du rugby à XV
}

\author{
Epidemiological review of injuries in Rugby Union
}

\author{
J.-F. Kaux ${ }^{\text {a, } *, b, c}$, M. Julia ${ }^{\text {d }}$, M. Chupin ${ }^{\text {c }}$, F. Delvaux ${ }^{\text {c }, ~ J .-L . ~ C r o i s i e r ~}{ }^{\text {b,c }}$, B. Forthomme ${ }^{\text {b,c }}$, \\ J.-M. Crielaard ${ }^{\mathrm{b}, \mathrm{c}}$, C. Le Goff ${ }^{\mathrm{b}}$, P. Durez ${ }^{\mathrm{e}}$, P. Ernst $^{\mathrm{a}}$, S. Guns ${ }^{\mathrm{a}}$, A. Laly ${ }^{\mathrm{a}}$ \\ ${ }^{a}$ Centre de formation de la Ligue belge francophone de rugby (LBFR), ADEPS du Blanc-Gravier, allée des Sports, P63, 4000 Liège, Belgique \\ ${ }^{\mathrm{b}}$ Service pluridisciplinaire de médecine et traumatologie du sport (SPORTS 2 ), CHU de Liège, avenue de l'Hôpital, B35, 4000 Liège, Belgique \\ ${ }^{\mathrm{c}}$ Département des sciences de la motricité, université de Liège, allée des Sports, P63, 4000 Liège, Belgique \\ ${ }^{\mathrm{d}}$ Commission médicale de la Fédération française de rugby (FFR), Fédération MPR Montpellier-Nîmes, hôpital Lapeyronie, CHRU de Montpellier, 34000 \\ Montpellier, France \\ ${ }^{\mathrm{e}}$ Commission médicale de la Fédération belge de rugby (FBR), 1000 Bruxelles, Belgique
}

Disponible sur Internet le 17 janvier 2014

\section{Résumé}

Le rugby est un sport en plein développement. Sport de contact par excellence, il est à l'origine d'un nombre important de blessures. Au rugby à XV, l'incidence des blessures s'élève de 30 à 91/1000 heures de match. Cette revue épidémiologique des blessures chez le rugbyman évoque leur localisation et leur nature, les causes, les moments des matches et de la saison auxquels elles se produisent, la position des joueurs et le type de terrain, ainsi que le temps d'interruption de la pratique à la suite d'une lésion.

(C) 2013 Elsevier Masson SAS. Tous droits réservés.

Mots clés : Blessures ; Rugby ; Épidémiologie ; Terrain

\begin{abstract}
Rugby is an increasingly popular sport. Full contact being a major characteristic of rugby, injuries are not uncommon. The incidence of injury in Rugby Union reaches 30-91 per 1,000 hours of game time. This epidemiological review of injuries in rugby players reports their localization, nature, causes, moment of occurrence in matches and seasonal trends as well as the influence of the player's position, field conditions, and duration of off time after injury.
\end{abstract}

(c) 2013 Elsevier Masson SAS. All rights reserved.

Keywords: Injuries; Rugby; Epidemiology; Field conditions

\section{Introduction}

Le rugby à XV est reconnu comme un sport de contact [1]. La lutte pour la possession du ballon est permanente [1] afin de marquer des points en utilisant ses capacités techniques et physiques.

Au rugby à XV, l'incidence des blessures s'élève de 30 à 91 , voire même 120 , pour 1000 heures de match selon les auteurs [2-7]. Des études de cohorte sur des joueurs écossais amateurs (jeunes et seniors) ont montré que l'incidence des blessures augmentait avec l'âge, allant de 3/1000 heures de jeu

\footnotetext{
* Auteur correspondant.

Adresse e-mail : jfkaux @chu.ulg.ac.be (J.-F. Kaux).
}

chez les moins de 16 ans, 18 à 24/1000 heures de match pour les 20-24 ans, 35/1000 heures de match pour les 25-29 ans et 10-21/1000 heures de match chez les plus de 35 ans $[3,8]$.

Les blessures occasionnées à l'entraînement sont plus rares (6/1000 heures d'entraînement) et atteignent généralement les membres inférieurs [7].

Lors de la dernière coupe du monde en Nouvelle-Zélande, l'incidence des blessures s'élevait à $89,1 / 1000$ heures de match et de 2,2/1000 heures d'entraînement [9].

\section{Localisation des blessures}

Le membre inférieur est le site le plus fréquemment atteint en match (30 à $55 \%$ des blessures), suivi de la tête et du rachis 
cervical (14 à $30 \%)$, puis du membre supérieur (15 à $20 \%)$ et enfin du tronc (10 à 14\%) [4,8,10-12]. Chez les professionnels, la tête (commotions incluses) est le plus souvent atteinte (25\%), suivie du genou (14-20\%), de la cuisse (13-19\%) et de la cheville $(11 \%)$, puis de l'épaule $(6,5 \%)$, de la main $(5,6 \%)$, de la jambe $(5,6 \%)$ et du pied $(3,5 \%)$ [5,13-16]. Chez les amateurs et les juniors, les blessures de l'épaule sont plus fréquentes ; néanmoins, le genou, la cuisse et la cheville restent parmi les sites les plus touchés [17-20].

\section{Types de blessures}

Chez les professionnels, plus de la moitié des lésions (55\%) sont des blessures fermées au niveau des tissus mous [4]. L'ensemble des blessures se répartissent en entorses ou déchirures capsulaires/ligamentaires (20-34\%), élongations ou déchirures musculotendineuses (20-29\%), plaies (12-27\%), contusions/hématomes (10-22\%), fractures (4-14\%), luxations/subluxations (4-10\%) et commotions cérébrales (3-10\%) $[4,7]$ (Tableau 1).

Les études concernant les semi-professionnels recensent des blessures musculaires (hématomes et contractures) plus répandues $(32,9 \%)$, tandis que les contusions $(20,2 \%)$ et les lésions articulaires $(15,6 \%)$ sont moins fréquentes $[11,21]$.

Tableau 1

Type de lésions et leur pourcentage en fonction du site anatomique (épaule, genou, cheville) $[14,15,19]$.

\begin{tabular}{|c|c|c|}
\hline $\begin{array}{l}\text { Site de la } \\
\text { blessure }\end{array}$ & Type de lésions & Pourcentage \\
\hline Épaule & $\begin{array}{l}\text { Disjonctions acromio-claviculaires } \\
\text { Lésions de la coiffe des rotateurs } \\
\text { Luxations/instabilités } \\
\text { Coups directs } \\
\text { Fractures }\end{array}$ & $\begin{array}{l}32 \% \\
23 \% \\
14 \% \\
12 \% \\
1 \%\end{array}$ \\
\hline Genou & $\begin{array}{l}\text { Entorses/ruptures du ligament collatéral } \\
\text { médial } \\
\text { Lésions méniscales/chondrales } \\
\text { Lésions fémoro-patellaires } \\
\text { Hématomes extra-articulaires } \\
\text { Entorses/ruptures du ligament croisé } \\
\text { postérieur } \\
\text { Entorses/ruptures du ligament croisé } \\
\text { antérieur } \\
\text { Bursopathies prépatellaires }\end{array}$ & $\begin{array}{l}28,9 \% \\
18,5 \% \\
12,3 \% \\
10,9 \% \\
9 \% \\
4,3 \% \\
1,4 \%\end{array}$ \\
\hline Cheville & $\begin{array}{l}\text { Lésions syndesmotiques tibiofibulaires } \\
\text { Lésions capsules articulaires } \\
\text { Entorses/ruptures du ligament collatéral } \\
\text { médial } \\
\text { Tendino-bursopathie calcanéenne } \\
\text { Hématomes } \\
\text { Conflits antérieurs ou postérieurs } \\
\text { Ruptures du tendon calcanéen } \\
\text { Fractures de malléole externe }\end{array}$ & $\begin{array}{l}41,4 \% \text { (grade } \\
1: 25 \% ; \\
\text { grade } 2: \\
14 \% ; \text { grade } \\
3: 2,4 \% \text { ) } \\
11 \% \\
11 \% \\
7,8 \% \\
7,8 \% \\
4,8 \% \\
4,8 \% \\
1,2 \% \\
1,2 \%\end{array}$ \\
\hline
\end{tabular}

Chez les joueurs amateurs, les lésions les plus courantes sont musculaires $(28,5 \%)$, puis articulaires $(17,2 \%)$ et les blessures ouvertes $(14,5 \%)$ [22]. Les fractures $(4,1 \%)$ et les luxations $(3,2 \%)$ sont beaucoup plus rares $[17,22,23]$.

Chez les plus jeunes, les lésions articulaires sont plus nombreuses [17,20].

Certaines blessures demeurent plus lourdes de conséquences. Au niveau cervical, des lésions peuvent présenter des répercussions neurologiques catastrophiques (tétraplégie) et des décès ont été rapportés [24,25]. L'incidence de ce type de blessures a diminué ces dernières années, certaines règles du jeu ayant été modifiées concernant l'entrée en mêlée afin d'assurer plus de sécurité aux joueurs [25]. Cependant, l'incidence des lésions cervicales aiguës avec déficit neurologique persistant et des traumatismes crâniens restait de 2 pour 100000 joueurs $(4,52 / 100000$ joueurs seniors et $0,24 / 100000$ joueurs juniors), selon une étude menée entre 2008 et 2011 en Afrique du Sud [26]. Ces données sont comparables à celles observées dans les autres sports de contact tels que le football américain [26]. Cependant, cette incidence de lésions dramatiques serait plus importante qu'en VTT [27] mais le serait moins qu'en équitation [28] ou en parapente [29]. Au niveau lombaire, des chocs violents, parfois concomitants à un problème discal préexistant, peuvent causer des cas de paraplégie [25].

\section{Causes des blessures}

La plupart des blessures (70\%) surviennent suite à un contact avec un autre joueur $[3,30]$. Le plaquage est la phase de jeu qui provoque le plus de blessures (entre 36 et 58\%), 23-29\% chez les joueurs plaqués et $13-27 \%$ chez les plaqueurs, la fréquence augmentant pour les joueurs les plus rapides [7,30,31]. D'autres études confirment la survenue lésionnelle plus élevée pour les joueurs subissant le plaquage [2,5,31-33]. L'incidence des lésions par plaquage se répartit comme suit : $39,1 \%$ pour les plaquages latéraux, $30,4 \%$ pour les plaquages de face, et $26,2 \%$ par derrière [34]. Chez les professionnels, 46 à $57 \%$ de blessures sont rapportées pour le joueur plaqué contre 21 à $42 \%$ pour le joueur plaqueur [32]. Ces fréquences deviennent, chez les semiprofessionnels, de $30 \%$ pour le joueur plaqué et $24 \%$ pour le joueur plaqueur [35]. Lors des blessures sur phase de plaquage, $22 \%$ des joueurs sont touchés à la nuque ou à la tête et la face, $17 \%$ au genou, $14 \%$ à l'épaule, $10 \%$ au bras et à la main, $8 \%$ à la cheville et $8 \%$ à la cuisse [3]. Les joueurs plaqués sont surtout blessés au membre inférieur (51\%), par rapport au membre supérieur $(15 \%)$ et à la tête $(17 \%)$, tandis que les plaqueurs sont surtout blessés au membre supérieur $(35 \%)$, puis à la tête $(28 \%)$ et au membre inférieur (27\%) [3]. D'autres causes rapportées telles que le ruck et mauls ( 15 à $36 \%$ ), les phases de course et de changement de direction (10\%) et les entrées en mêlées (1 à $7 \%$ ) provoquent moins de lésions [5,7,10,12,21,30,36], mais peuvent être potentiellement plus graves, notamment concernant les entrées en mêlées [24-26].

Au niveau de la cheville, la cause des blessures reste pour la moitié $(53 \%)$ une action de contact; $35 \%$ sont des incidents de non-contact avec un autre joueur et $12 \%$ sont dus à des mécanismes inconnus [15]. 
Concernant l'épaule, la majorité des lésions en match arrive suite à un contact avec un autre joueur (97\%) [19], préférentiellement durant une phase de plaquage $(65 \%: 40 \%$ pour les plaqueurs, $25 \%$ pour les plaqués). Une grande proportion des luxations d'épaule et disjonctions acromio-claviculaires se produit lors d'un plaquage [19].

Soixante-douze pour cent des blessures de genou en match arrivent pendant une phase de contact, $22 \%$ de non-contact et $6 \%$ de mécanisme inconnu [14]. Le plaquage entraîne la plus grande cause de blessures de genou [14].

Les lésions surviennent également suite à des comportements irréguliers, rarement pénalisés par l'arbitre, dans une proportion variant entre 14 et $16 \%$ selon les auteurs $[11,22]$. De toutes les blessures, $5 \%$ sont dues à des actions fautives [3,5]. Les lésions au niveau de la tête (incluant la face et les yeux) et les contusions musculaires sont plus fréquentes dans les situations irrégulières $[11,37]$.

Lors de la dernière coupe du monde, les plaquages ont été responsables de l'essentiel des lésions en match (45\%) et les activités de contact lors des entraînements [9].

\section{Blessures et charge d'entraînement}

Gabbett souligne une corrélation significative entre la charge d'entraînement et les blessures [38]. Autrement dit, plus la charge est élevée, plus le risque de blessure l'est aussi. D'autres recherches portant sur la ligue de rugby ont largement reconnu que si l'intensité, la durée et la charge d'une séance d'entraînement ou d'un match augmentent, il en va de même pour l'incidence des blessures [21].

Il semble également qu'une mauvaise condition physique ainsi qu'une faible charge d'entraînement contribuent au risque de blessure. Une étude a mis en évidence que les joueurs ayant une mauvaise condition physique et ceux qui avaient effectué moins de 18 semaines d'entraînement avant de se blesser avaient un risque accru de récidive lésionnelle [35].

\section{Moments du match et de la saison auxquels les blessures surviennent}

Dans une étude concernant les hommes et les femmes, $46 \%$ des blessures se produisent durant la première mi-temps et $40 \%$ durant la seconde; pour les $14 \%$ restant, l'information n'a pas été donnée [11]. Pour d'autres auteurs, il y a plus de blessures durant la seconde période (entre 55 et $70 \%$ ) que durant la première (entre 30 et $45 \%$ ). De nombreux médecins de terrain observent que le nombre de blessés augmente au fur et à mesure du match $[3,5,11,23,33,37,39]$, mais il semblerait que les périodes les plus critiques soient le deuxième et surtout le quatrième quart temps [30].

Une plus faible proportion des blessures de cheville arrive durant la première mi-temps $(42 \%)$ par rapport à la seconde $(58 \%)$, la majorité de ces blessures se déroulant pendant les 20 dernières minutes de jeu $(35 \%)$ [15]. Pour le tendon calcanéen, les blessures se produisent avec une plus grande incidence lors de la première mi-temps alors que les blessures du
Tableau 2

Synthèse du nombre de lésions aux postes avants et arrières en fonction de l'action et de la partie du corps atteinte $[11,14,15,19,21,23,36,39]$.

\begin{tabular}{lrc}
\hline & Avants & Arrières \\
\hline Actions & & \\
Actions fautives & $7 \%$ & $6 \%$ \\
Contacts avec un autre joueur & $70 \%$ & $61 \%$ \\
Site de la blessure & & \\
Membre inférieur & $54 \%$ & $65 \%$ \\
$\quad$ Genou & $10 \%$ & $17 \%$ \\
$\quad$ Ischio-jambiers & $6 \%$ & $15 \%$ \\
Membre supérieur & $19 \%$ & $14 \%$ \\
$\quad$ Épaule & $10 \%$ & $10 \%$ \\
Tronc & $16 \%$ & $9 \%$ \\
Tête & $10 \%$ & $12 \%$ \\
\hline
\end{tabular}

ligament collatéral latéral de cheville prédominent durant la seconde mi-temps [15].

Concernant l'épaule, l'incidence des blessures augmente au fur et à mesure du match [19].

Les blessures de genou se produisent plus souvent durant la seconde mi-temps $(58 \%)$ et principalement durant les 20 dernières minutes de match $(32 \%)$, avec une incidence plus basse au début du jeu [14].

L'incidence des blessures aurait tendance à augmenter progressivement durant la saison, surtout pour les clubs participant à de nombreuses compétitions (championnat, coupes. . .) [21,23].

\section{Position du joueur : postes avants/arrières}

Lors d'un match, chaque équipe effectue une moyenne de 300 plaqués. Les avants sont exposés à une moyenne de 55 collisions physiques contre 29 pour les arrières [40].

L'incidence des blessures (Tableau 2) sur actions fautives reste similaire pour les joueurs à l'avant et ceux à l'arrière [37]. L'incidence globale des lésions dues à un contact est plus importante pour les avants que pour les arrières [5-7]. Le plaquage entraîne davantage de blessures pour les arrières et l'incidence de blessures pendant un ruck ou un maul est significativement plus grande pour les avants [3]. La sévérité des blessures en match ne diffère pas significativement selon les postes occupés. Cependant, les auteurs ne semblent pas du même avis quant au taux d'incidence des blessures [11,14,15,19,21,23,36,39].

L'analyse de l'incidence des blessures lors de la dernière coupe du monde a permis d'observer que les arrières s'étaient davantage blessés $(93,8 / 100000$ heures de match) que les avants (85,0/100 000 heures de match) et qu'ils présentaient des lésions plus graves durant les matches, mais moins graves lors de l'entraînement [9].

\section{Position du joueur : positions détaillées}

Sur cette question, les avis sont partagés. Pour certains, les deuxièmes lignes auraient une incidence plus grande de blessures que les autres positions, tandis que les 9,10 et 12 présentent une incidence plus faible de blessures [11]. Pour d'autres, les ailiers occupent la position la plus souvent atteinte $(21,6 \%)$, 
suivie des centres $(18,9 \%)$ [33]. Pour d'autres encore, ce sont les talonneurs et les demis d'ouverture qui ont la plus grande incidence de blessures alors que les deuxièmes lignes droits et troisièmes lignes ailes subissent les blessures les plus sévères [5].

En terme de remplacement, les troisièmes lignes ailes suivis des piliers sont les positions les plus souvent remplacées pour cause de blessure [12,37]; au niveau des arrières, ce sont les ailiers et les centres qui prédominent. L'incidence de remplacement est fort similaire pour tous les arrières $[12,37]$.

\section{Temps d'absence}

Les blessures sont classées en 3 catégories en fonction du temps d'arrêt de la compétition. Les blessures mineures sont celles pour lesquelles la durée d'absence est de moins d'une semaine. Lorsque le joueur est absent d'une à trois semaines, la blessure est considérée comme modérée. Après trois semaines d'arrêt de la compétition, la lésion est dite sévère. La plupart des blessures sont considérées comme mineures (64-82\%), les blessures modérées (10-18\%) et sévères (8-22\%) s'avèrent moins fréquentes $[5,7,12,17,36]$.

Les blessures au niveau du genou entraînent le plus grand nombre de jours d'absence (21\%), suivies par les luxations de l'épaule (15\%); les lésions de cuisse (11\%) et de cheville (10\%) sont moins lourdes de conséquences $[3,14,15]$.

Concernant les blessures de genou, les lésions du ligament croisé antérieur entraînent $29 \%$ des jours d'absence, $26 \%$ pour le ligament collatéral médial et $21 \%$ pour des lésions chondroméniscales [14].

Pour les blessures d'épaule, les luxations représentent $42 \%$ des jours d'absence [19]. Les lésions de l'articulation acromio-claviculaire et de la coiffe des rotateurs sont un peu moins sévères et représentent chacune environ $20 \%$ des jours d'absence du joueur [19].

Au niveau des blessures musculaires des ischio-jambiers, les blessures étant classées dans les 3 catégories ci-dessus, $37 \%$ sont reconnues comme mineures, $37 \%$ comme modérées et $26 \%$ comme sévères [39].

Les lésions du ligament collatéral latéral (29\%) et du tendon calcanéen, rupture incluse $(27 \%)$, représentent le plus de temps d'absence pour blessure au niveau de la cheville [15].

\section{Type de terrain}

Les terrains artificiels sont une alternative rentable au gazon naturel car ils peuvent être utilisés par tous les temps et pour de nombreuses activités. Les gazons artificiels évoluent depuis 50 ans et essaient de se rapprocher de plus en plus du gazon naturel. Il existe 3 générations de gazons artificiels et chacun possède ses propres caractéristiques :

- la première génération, apparue fin des années 1960, est composée de fibres de nylon très courtes. Elle est caractérisée par une force de friction élevée, une raideur et une rétention de chaleur plus élevées que pour les surfaces naturelles ;
Tableau 3

Nombre de blessures selon le type de terrain [41].

\begin{tabular}{|c|c|c|}
\hline & Herbe & Synthétique \\
\hline \multicolumn{3}{|l|}{ Sévérité } \\
\hline Mineure & $10,6 / 1000 \mathrm{~h}$ de jeu & $11,8 / 1000 \mathrm{~h}$ de jeu \\
\hline Modérée & $10,6 / 1000 \mathrm{~h}$ de jeu & $16,9 / 1000 \mathrm{~h}$ de jeu \\
\hline Sévère & $5,8 / 1000 \mathrm{~h}$ de jeu & $9,6 / 1000 \mathrm{~h}$ de jeu \\
\hline \multicolumn{3}{|l|}{ Site de la blessure } \\
\hline Tête/nuque & $25 \%$ & $19,2 \%$ \\
\hline Membre supérieur & $32,1 \%$ & $17,3 \%$ \\
\hline Tronc & $3,6 \%$ & $5,8 \%$ \\
\hline Membre inférieur & $39,3 \%$ & $57,7 \%$ \\
\hline \multicolumn{3}{|l|}{ Actions } \\
\hline Contact entre joueurs & $70,4 \%$ & $79,6 \%$ \\
\hline Non-contact & $29,6 \%$ & $20,4 \%$ \\
\hline
\end{tabular}

- la deuxième génération est apparue au début des années 1980. Les fibres qui la composent sont plus longues et le rembourrage est plus épais ;

- la troisième génération date de la fin des années 1990 et se caractérise par des fibres plus longues, remplies de caoutchouc synthétique absorbant mieux les chocs.

Lorsque le match se déroule sur un terrain en herbe, 26,9 blessures par 1000 heures de jeu sont recensées. Sur un terrain de type synthétique, il est de 38,2 par 1000 heures de jeu [41] (Tableau 3). La plupart des blessures se produisant sur le gazon artificiel serait associée à l'adaptation des mouvements sur des surfaces dures, avec une augmentation de l'activité musculaire excentrique et une modification des habitudes de déplacement dans le genou et la cheville [42]. De plus, avec des surfaces artificielles, les forces de traction sont supérieures et les joueurs peuvent acquérir une plus grande vitesse [42].

Enfin, en plus de la surface, il faut également tenir compte des conditions météorologiques. Par exemple, au football américain sur gazon naturel le taux de blessures semble être plus élevé en condition sèche que sur sol mouillé [43]. La même tendance a été observée avec les gazons artificiels de première génération [44].

\section{Conclusion}

Le rugby à XV est un sport en plein développement. Sport de contact par excellence, il est à l'origine d'un nombre important de traumatismes. Le recensement épidémiologique de blessures lors de la pratique de ce sport (par poste, moment du match, type de terrain. ..) est nécessaire pour mettre en évidence des facteurs de risque de lésions et pouvoir instaurer des protocoles de préventions spécifiques [45]. Cependant, aucune différence n'a pu être observée concernant l'incidence, la cause et la sévérité des blessures entre les 2 dernières coupes du monde de 2007 et 2011 [9]. 


\section{Déclaration d'intérêts}

Les auteurs déclarent ne pas avoir de conflits d'intérêts en relation avec cet article.

\section{Références}

[1] International Rugby Board. Les règles du jeu et la charte du jeu. Dublin: International Rugby Board; 2013. p. 216.

[2] Targett SG. Injuries in professional rugby union. Clin J Sport Med $1998 ; 8: 280-5$.

[3] Garraway WM, et al. Impact of professionalism on injuries in rugby union. Br J Sports Med 2000;34:348-51.

[4] Bathgate A, et al. A prospective study of injuries to elite Australian rugby union players. Br J Sports Med 2002;36:265-9 [discussion 269].

[5] Brooks JH, et al. Epidemiology of injuries in English professional rugby union: part 1 match injuries. Br J Sports Med 2005;39:757-66.

[6] Brooks JH, et al. Epidemiology of injuries in English professional rugby union: part 2 training injuries. Br J Sports Med 2005;39:767-75.

[7] Kaplan KM, et al. Rugby injuries: a review of concepts and current literature. Bull NYU Hosp Jt Dis 2008;66:86-93.

[8] Garraway M, Macleod D. Epidemiology of rugby football injuries. Lancet 1995;345(8963):1485-7.

[9] Fuller CW, Sheerin K, Targett S. Rugby World Cup 2011: International Rugby Board Injury surveillance study. Br J Sports Med 2013;47(18):1184-91.

[10] Lee AJ, Garraway WM. Epidemiological comparison of injuries in school and senior club rugby. Br J Sports Med 1996;30:213-7.

[11] Bird YN, et al. The New Zealand Rugby Injury and Performance Project: V. Epidemiology of a season of rugby injury. Br J Sports Med 1998;32:319-25.

[12] Bottini E, et al. Incidence and nature of the most common rugby injuries sustained in Argentina (1991-1997). Br J Sports Med 2000;34:94-7.

[13] Gerrard DF, Waller AE, Bird YN. The New Zealand Rugby Injury and Performance Project: II. Previous injury experience of a rugby-playing cohort. Br J Sports Med 1994;28:229-33.

[14] Dallalana RJ, et al. The epidemiology of knee injuries in English professional rugby union. Am J Sports Med 2007;35:818-30.

[15] Sankey RA, et al. The epidemiology of ankle injuries in professional rugby union players. Am J Sports Med 2008;36:2415-24.

[16] Pearce CJ, et al. The epidemiology of foot injuries in professional rugby union players. Foot Ankle Surg 2011;17:113-8.

[17] Junge A, et al. Injuries in youth amateur soccer and rugby players - comparison of incidence and characteristics. Br J Sports Med 2004;38:168-72.

[18] Yard EE, Comstock RD. Injuries sustained by rugby players presenting to United States emergency departments, 1978 through 2004. J Athl Train 2006;41:325-31

[19] Headey J, Brooks JH, Kemp SP. The epidemiology of shoulder injuries in English professional rugby union. Am J Sports Med 2007;35:1537-43.

[20] Gabbett TJ. Incidence of injury in junior rugby league players over four competitive seasons. J Sci Med Sport 2008;11:323-8.

[21] Gabbett TJ. Incidence of injury in semi-professional rugby league players. Br J Sports Med 2003;37:36-43 [discussion 43-4].

[22] Gabbett TJ. Severity and cost of injuries in amateur rugby league: a case study. J Sports Sci 2001;19:341-7.
[23] Gabbett TJ. Incidence, site, and nature of injuries in amateur rugby league over three consecutive seasons. Br J Sports Med 2000;34:98-103.

[24] Quarrie KL, Cantu RC, Chalmers DJ. Rugby union injuries to the cervical spine and spinal cord. Sports Med 2002;32:633-53.

[25] Fuller CW. Catastrophic injury in rugby union: is the level of risk acceptable? Sports Med 2008;38:975-86.

[26] Brown JC, et al. The incidence of rugby-related catastrophic injuries (including cardiac events) in South Africa from 2008 to 2011: a cohort study. BMJ Open 2013:3(2).

[27] Dodwell ER, et al. Spinal column and spinal cord injuries in mountain bikers: a 13-year review. Am J Sports Med 2010;38:1647-52.

[28] Loder RT. The demographics of equestrian-related injuries in the United States: injury patterns, orthopedic specific injuries, and avenues for injury prevention. J Trauma 2008;65:447-60.

[29] Schulze W, et al. Injury prophylaxis in paragliding. Br J Sports Med 2002;36:365-9.

[30] Fuller CW, et al. Contact events in rugby union and their propensity to cause injury. Br J Sports Med 2007;41:862-7 [discussion 867].

[31] Gabbett TJ, Ullah S, Finch CF. Identifying risk factors for contact injury in professional rugby league players - application of a frailty model for recurrent injury. J Sci Med Sport 2012;15:496-504.

[32] Stephenson S, Gissane C, Jennings D. Injury in rugby league: a four-year prospective survey. Br J Sports Med 1996;30:331-4.

[33] Nicol A, et al. Rugby union injuries in Scottish schools. J Public Health (Oxf) 2011;33:256-61.

[34] Wilson BD, et al. The nature and circumstances of tackle injuries in rugby union. J Sci Med Sport 1999;2:153-62.

[35] Gabbett TJ, Domrow N. Risk factors for injury in subelite rugby league players. Am J Sports Med 2005;33:428-34.

[36] Brooks JH, et al. A prospective study of injuries and training amongst the England 2003 Rugby World Cup squad. Br J Sports Med 2005;39: 288-93.

[37] Sharp JC, Murray GD, Macleod DA. A unique insight into the incidence of rugby injuries using referee replacement reports. Br J Sports Med 2001;35:34-7

[38] Gabbett TJ. Influence of training and match intensity on injuries in rugby league. J Sports Sci 2004;22:409-17.

[39] Brooks JH, et al. Incidence, risk, and prevention of hamstring muscle injuries in professional rugby union. Am J Sports Med 2006;34: 1297-306.

[40] Gabbett TJ, Jenkins DG. Relationship between training load and injury in professional rugby league players. J Sci Med Sport 2011;14:204-9.

[41] Fuller CW, Clarke L, Molloy MG. Risk of injury associated with rugby union played on artificial turf. J Sports Sci 2010;28:563-70.

[42] Pérez-Soriano P, et al. Biomechanical factors to be taken into account to prevent injuries and improve sporting performance on artificial turf. Sport Exerc 2009;4:78-92.

[43] Andresen BL, Hoffman MD, Barton LW. High school football injuries: field conditions and other factors. Wis Med J 1989;88:28-31.

[44] Adkison JW, Requa RK, Garrick JG. Injury rates in high school football. A comparison of synthetic surfaces and grass fields. Clin Orthop Relat Res 1974:131-6.

[45] van Mechelen W, Hlobil H, Kemper HC. Incidence, severity, aetiology and prevention of sports injuries. A review of concepts. Sports Med 1992;14:82-99. 